\title{
The FERRUM project: laboratory-measured transition probabilities for $\mathrm{Cr}{ }^{\star}{ }^{\star}$
}

\author{
J. Gurell ${ }^{1}$, H. Nilsson ${ }^{2}$, L. Engström ${ }^{3}$, H. Lundberg ${ }^{3}$, R. Blackwell-Whitehead ${ }^{2}$, K. E. Nielsen ${ }^{4,5}$, and S. Mannervik ${ }^{1}$ \\ 1 Department of Physics, Stockholm University, AlbaNova University Center, 10691 Stockholm, Sweden \\ e-mail: jonas.gurell@fysik.su.se \\ 2 Lund Observatory, Lund University, Box 43, 22100 Lund, Sweden \\ 3 Atomic Physics, Department of Physics, Lund University, Box 118, 22100 Lund, Sweden \\ 4 Catholic University of America, Washington, DC 20064, USA \\ 5 Astrophysics Science Division, Code 667, Goddard Space Flight Center, Greenbelt, MD 20771, USA
}

Received 16 November 2009 / Accepted 15 December 2009

\section{ABSTRACT}

\begin{abstract}
Aims. We measure transition probabilities for Cr II transitions from the $z^{4} \mathrm{H}_{J}, z^{2} \mathrm{D}_{J}, y^{4} \mathrm{~F}_{J}$, and $y^{4} \mathrm{G}_{J}$ levels in the energy range 63000 to $68000 \mathrm{~cm}^{-1}$.

Methods. Radiative lifetimes were measured using time-resolved laser-induced fluorescence from a laser-produced plasma. In addition, branching fractions were determined from intensity-calibrated spectra recorded with a UV Fourier transform spectrometer. The branching fractions and radiative lifetimes were combined to yield accurate transition probabilities and oscillator strengths. Results. We present laboratory measured transition probabilities for $145 \mathrm{Cr}$ II lines and radiative lifetimes for $14 \mathrm{Cr}$ II levels. The laboratory-measured transition probabilities are compared to the values from semi-empirical calculations and laboratory measurements in the literature.
\end{abstract}

Key words. atomic data - line: identification - methods: laboratory - techniques: spectroscopic

\section{Introduction}

Spectral analysis of astrophysical objects depends on the availability of accurate laboratory data including radiative lifetimes and transition probabilities. Lines of $\mathrm{Cr}$ II are observed in a broad range of stellar and nebular spectra (e.g. Merrill 1951; Shevchenko 1994; Andrievsky et al. 1994), and accurate Cr data are required for stellar abundance studies (Babel \& Lanz 1992; Dimitrijević et al. 2007). In particular, several chemically peculiar stars show unexpectedly high abundances of $\mathrm{Cr}$ (Rice \& Wehlau 1994; López-García et al. 2001).

Radiative lifetimes in $\mathrm{Cr}$ II have been measured with the beam-foil technique by Pinnington et al. (1973) and Engman et al. (1975) and with the time-resolved laser-induced fluorescence (TRLIF) technique by Schade et al. (1990), Pinnington et al. (1993), and Nilsson et al. (2006). In addition, branching fraction $(B F)$ measurements were combined with radiative lifetimes to yield transition probabilities (Bergeson \& Lawler 1993; Spreger et al. 1994; Gonzalez et al. 1994; Nilsson et al. 2006), and oscillator strengths were measured by Musielok \& Wujec (1979), Goly \& Weniger (1980) and Wujec \& Weniger (1981) using a wall-stabilized arc. Semi-empirical oscillator strengths have been calculated by Kurucz (1988) using the Cowan code, by Luke (1988) using the R matrix method, and by Raasen \& Uylings (1997) using the orthogonal operator method.

Several studies have found that the stellar chromium abundance determined from $\mathrm{Cr} \mathrm{I}$ lines is significantly different to

* Tables 2 and 3 are only available in electronic form at the CDS via anonymous ftp to cdsarc.u-strasbg. fr (130.79.128.5) or via http://cdsweb.u-strasbg.fr/cgi-bin/qcat?J/A+A/511/A68 the abundance using Cr II lines (McWilliam et al. 1995; Sobeck et al. 2007; Lai et al. 2008). This difference is greater than the uncertainty in the stellar observations and measured oscillator strengths. Furthermore, the difference increases as the metallicity of the star decreases. Sobeck et al. (2007) indicate that one possible explanation of this discrepancy could be non-LTE effects not included in the stellar model. Sobeck et al. (2007) propose that, in order to resolve this issue additional laboratory investigations of chromium should focus on weak branches of $\mathrm{Cr}$ II and that the $\mathrm{Cr}$ abundance should be reanalyzed with a three-dimensional hydrodynamical model. In addition, the need for more laboratory measured $\mathrm{Cr}$ II transition probabilities is discussed by Wallace \& Hinkle (2009).

In this paper we present transition probabilities for 145 lines in Cr II from 14 upper levels, see the partial energy level diagram of $\mathrm{Cr}$ II in Fig. 1. The lifetimes of the upper levels have been measured with the TRLIF technique.

\section{Laboratory measurements}

The lifetime of an upper state $i$ can be written as

$\tau_{i}=1 / \sum_{k} A_{i k}$

where $A_{i k}$ is the transition probability of a line from upper level $i$ to lower level $k$. The $B F$ of the line is defined as

$B F_{i k}=A_{i k} / \sum_{k} A_{i k}=I_{i k} / \sum_{k} I_{i k}$ 


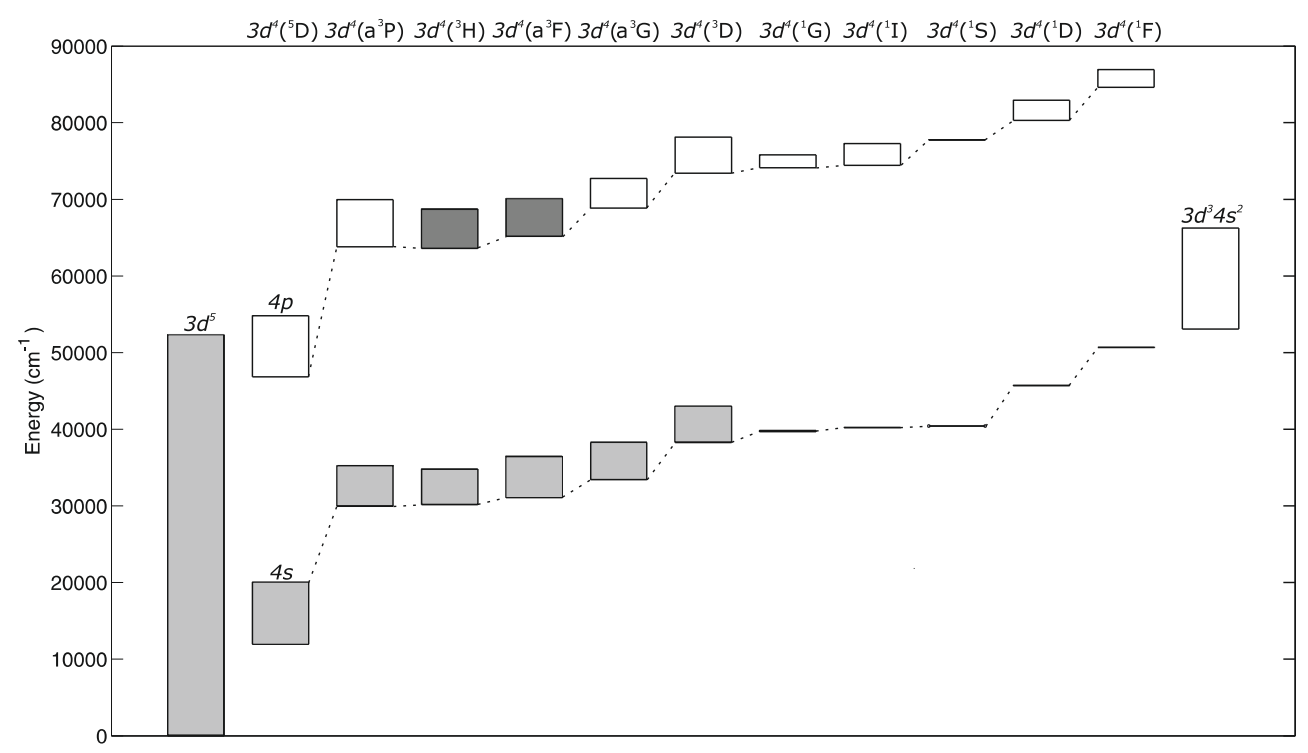

Fig. 1. Partial energy level diagram of Cr II (Ralchenko et al. 2009). Only terms belonging to the $3 d^{5}, 3 d^{4} 4 s, 3 d^{4} 4 p$, and $3 d^{3} 4 s^{2}$ configurations are shown and ordered by their respective parent term shown above the diagram. The investigated upper levels are shown as dark grey boxes and the lower levels to which they decay are shown as light grey boxes.

where $I_{i k}$ is the measured intensity corrected for the instrumental response. Combining these two equations gives the transition probabilities as

$A_{i k}=B F_{i k} / \tau_{\mathrm{i}}$.

The following subsections describe the measurements of the lifetimes and $B F$ s.

\subsection{Radiative lifetimes}

We measured lifetimes for 14 odd parity levels in $\mathrm{Cr}$ II belonging to the $3 d^{4} 4 p$ configuration. The lifetimes were measured using the TRLIF technique at the Lund High Power Laser Facility. This technique has been described in detail in the literature (see Bergström et. al. 1988; Xu et al. 2003), so only a brief description is given here.

A laser-produced "plasma-cone" containing $\mathrm{Cr}$ atoms and ions in metastable levels was created by focusing a Nd:YAG laser (Continuum Surelite) onto a target of pure $\mathrm{Cr}$. $\mathrm{Cr}^{+}$ions in metastable states were excited to levels of opposite parity using a pump laser and the fluorescence from the excited levels was recorded as a function of time. The excitation pulses were created by pumping a Continuum Nd-60 dye laser with a Nd:YAG Continuum NY-82 laser. The pulses from the Nd:YAG pump laser were shortened from 10 to $1.5 \mathrm{~ns}$ using stimulated Brillouin scattering. The Nd-60 dye laser used a DCM dye to produce light between 6000 and $6700 \AA$. A broader wavelength coverage was achieved by using nonlinear effects in KDP and BBO crystals and Raman shifts in a $\mathrm{H}_{2}$ cell.

A $1 / 8 \mathrm{~m}$ monochromator was used to select the observable fluorescence wavelength. The fluorescence signal was recorded with a micro-channel plate photomultiplier tube with a rise time of $0.2 \mathrm{~ns}$. The shape of the excitation pulse was measured with the same system as the fluorescence signal. The lifetimes were extracted by fitting the fluorescence data with a single exponential convoluted with the shape of the laser pulse. Each lifetime curve was averaged over 1000 laser shots, and the final lifetimes given in Table 1 are averages of at least 10 lifetime curves. The uncertainties in the lifetimes include both statistical and systematic errors.

\subsection{Branching fractions}

The $B F$ s were measured from spectra recorded with the Chelsea Instrument FT500 UV FT spectrometer at Lund Observatory. The light source was a Penning discharge lamp with pure $\mathrm{Cr}$ cathodes and operated with $\mathrm{Ne}$ as buffer gas. The light source was operated at a current between 0.8 and $1.3 \mathrm{~A}$, and with at a carrier gas pressure of 40 mTorr. The Penning discharge lamp provides an intensity stable emission spectrum over several hours enabling high signal-to-noise (S/N) spectra to be recorded. Figure 2 shows part of the observed spectrum.

Three separate spectral regions were recorded to cover the wavenumber region between 20000 and $52000 \mathrm{~cm}^{-1}$. The spectra were intensity-calibrated with standard lamps with known spectral radiances and by $\mathrm{Cr}$ II $B F$ s previously measured by Nilsson et al. (2006). A continuous deuterium $\left(\mathrm{D}_{2}\right)$ lamp was used in the wavenumber range $32000-52000 \mathrm{~cm}^{-1}$. A tungsten strip lamp was used in the wavenumber range $20000-28000 \mathrm{~cm}^{-1}$ and the $28000-32000 \mathrm{~cm}^{-1}$ wavenumber region was calibrated using previously intensity-calibrated Cr II $B F$ s from the data of Nilsson et al. (2006). The calibration was performed with a software routine implemented in the program XGremlin (Nave et al. 1997). In addition, the spectra were wavenumber-calibrated using an average of nine unblended Ne II lines, which have been measured with high accuracy and suggested as suitable transitions for wavenumber calibration by Öberg (2007).

However, the wavelengths and wavenumbers in Tables 2 and 3 are Ritz values determined from the energy levels in Ralchenko et al. (2009).

The spectral lines were fitted with Voigt profiles to determine the integrated intensity using the commercially available software PeakFit. The uncertainty in the integrated intensity is determined from the standard deviation in the fitted values and the $\mathrm{S} / \mathrm{N}$ of the lines. The majority of the fitted Cr II line profiles were unblended. However, a small number of $\mathrm{Cr}$ II lines were partially blended with other lines. The blended features were identified, and a fit of both line profiles was performed when possible. The blended Cr II lines are given with larger uncertainties in the integrated intensity. 
Table 1. Lifetimes in Cr II.

\begin{tabular}{|c|c|c|c|c|c|c|c|c|c|c|}
\hline \multirow[b]{2}{*}{ Configuration } & \multirow[b]{2}{*}{ Term } & \multirow[b]{2}{*}{$E\left(\mathrm{~cm}^{-1}\right)$} & \multicolumn{8}{|c|}{$\tau(\mathrm{ns})$} \\
\hline & & & This work & $C \& B^{a}$ & $W T^{b}$ & $P^{c}$ & $W E^{d}$ & $E^{e}$ & $L^{f}$ & $R \& U^{g}$ \\
\hline$\left(a^{3} \mathrm{H}\right) 4 \mathrm{p}$ & $z^{4} \mathrm{H}_{7 / 2}^{\mathrm{o}}$ & 63600 & $4.4(4)$ & $2.1(4)^{h}$ & $3.3^{h}$ & $4.0(4)^{h}$ & $5.3^{h, i}$ & & 3.3 & 4.4 \\
\hline$\left(a^{3} \mathrm{H}\right) 4 \mathrm{p}$ & $z^{4} \mathrm{H}_{9 / 2}^{\mathrm{o}}$ & 63706 & $4.4(4)$ & $2.1(4)^{h}$ & $3.3^{h}$ & $4.0(4)^{h}$ & $5.3^{h, i}$ & & 3.3 & 4.4 \\
\hline$\left(a^{3} \mathrm{H}\right) 4 \mathrm{p}$ & $z^{4} \mathrm{H}_{11 / 2}^{\mathrm{o}}$ & 63849 & $4.2(3)$ & $2.1(4)^{h}$ & $3.3^{h}$ & $4.0(4)^{h}$ & $5.3^{h, i}$ & $5.0(5)$ & 3.3 & 4.4 \\
\hline$\left(a^{3} \mathrm{H}\right) 4 \mathrm{p}$ & $z^{4} \mathrm{H}_{13 / 2}^{\mathrm{o}}$ & 64031 & $4.2(3)$ & $2.1(4)^{h}$ & $3.3^{h}$ & $4.0(4)^{h}$ & $5.3^{h, i}$ & $4.7(5)$ & 3.3 & 4.3 \\
\hline$\left(a^{3} \mathrm{~F}\right) 4 \mathrm{p}$ & $y^{4} \mathrm{~F}_{5 / 2}^{\mathrm{o}}$ & 67012 & $3.7(4)$ & & & & & & & 4.3 \\
\hline$\left(a^{3} \mathrm{~F}\right) 4 \mathrm{p}$ & $y^{4} \mathrm{~F}_{3 / 2}^{\mathrm{o}}$ & 67070 & $3.9(5)$ & & & & & & & 4.5 \\
\hline$\left(a^{3} \mathrm{~F}\right) 4 \mathrm{p}$ & $y^{4} \mathrm{~F}_{7 / 2}^{\mathrm{o}}$ & 67393 & $2.9(2)$ & & & & & & & 2.9 \\
\hline$\left(a^{3} \mathrm{~F}\right) 4 \mathrm{p}$ & $y^{4} \mathrm{~F}_{9 / 2}^{\mathrm{o}}$ & 67448 & $2.9(2)$ & & & & & & & 2.9 \\
\hline$\left(a^{3} \mathrm{H}\right) 4 \mathrm{p}$ & $y^{4} \mathrm{G}_{7 / 2}^{\mathrm{o}}$ & 67333 & $2.6(2)$ & & & $3.4(2)^{h h}$ & & & & 2.6 \\
\hline$\left(a^{3} \mathrm{H}\right) 4 \mathrm{p}$ & $y^{4} \mathrm{G}_{5 / 2}^{\mathrm{o}}$ & 67344 & $2.6(2)$ & & & $3.4(2)^{h}$ & & & & 2.5 \\
\hline$\left(a^{3} \mathrm{H}\right) 4 \mathrm{p}$ & $y^{4} \mathrm{G}_{9 / 2}^{\mathrm{o}}$ & 67353 & $2.6(2)$ & & & $3.4(2)^{h h}$ & & & & 2.5 \\
\hline$\left(a^{3} \mathrm{H}\right) 4 \mathrm{p}$ & $y^{4} \mathrm{G}_{11 / 2}^{\mathrm{o}}$ & 67369 & $2.7(2)$ & & & $3.4(2)^{h}$ & & & & 2.5 \\
\hline$\left(a^{3} \mathrm{~F}\right) 4 \mathrm{p}$ & $z^{2} \mathrm{D}_{3 / 2}^{\mathrm{o}}$ & 67379 & $3.1(3)$ & & & & & & & 3.3 \\
\hline$\left(a^{3} \mathrm{~F}\right) 4 \mathrm{p}$ & $z^{2} \mathrm{D}_{5 / 2}^{\mathrm{o}}$ & 67387 & $3.1(3)$ & & & & & & & 3.4 \\
\hline
\end{tabular}

Notes. ${ }^{(a)}$ Corliss \& Bozman (1962) (Intensity measurement). ${ }^{(b)}$ Warner (1967) (Calculated). ${ }^{(c)}$ Pinnington et al. (1973) (Beam-foil measurement). ${ }^{(d)}$ Warner (1967) (Emission measurement). ${ }^{(e)}$ Engman et al. (1975) (Beam-foil measurement. ${ }^{(f)}$ Luke (1988) (Calculated). ${ }^{\left({ }^{g}\right)}$ Raasen \& Uylings (1997) (Calculated). ${ }^{(h)}$ Mean lifetime. ${ }^{(i)}$ Upper limit .

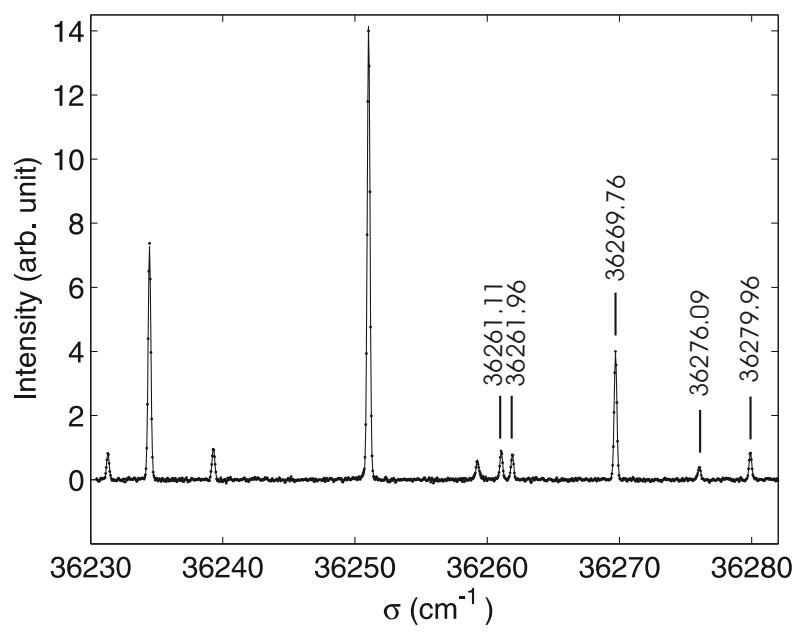

Fig. 2. A section of the FT spectrum showing five Cr II lines indicated by their respective wavenumber.

\section{Results}

Our experimental lifetimes are compared to previous measurements and calculations in Table 1 . Table 2 shows our laboratory measured $B F \mathrm{~s}, A$-values and semi-empirical $B F \mathrm{~s}$. The complete line list for all $\mathrm{Cr}$ II lines measured in this paper with comparisons to semi-empirical log $g f$ values in the literature is shown in Table 4. A comparison between our measured $A$-values and values presented in the literature is shown in Table 4.

\subsection{Lifetimes}

In Table 1 we report radiative lifetimes for 14 levels in $\mathrm{Cr}$ II compared with values from the literature. Our experimental lifetimes of the $z^{4} \mathrm{H}$ term agree, within the uncertainties, with the beamfoil measurements by Pinnington et al. (1973) and by Engman et al. (1975), and the experimental lifetimes of Warner (1967).
There is a good agreement between our lifetimes and the values from Raasen \& Uylings (1997); however, the calculated values by Warner (1967) and Luke (1988) are shorter than our lifetimes by approximately four standard deviations.

There is a a three-sigma difference between our lifetimes for the $y^{4} \mathrm{G}$ term and the measured values of Pinnington et al. (1973). The difference may come from the improved wavelength resolution of our measurements. Pinnington et al. (1973) were not able to resolve the four individual levels $y^{4} \mathrm{G}$ and measured an average lifetime determined from a blend of transitions from these levels. Pinnington et al. (1973) also states that the assignment of the transition from the $y^{4} \mathrm{G}$ term at $2700 \AA$ is one of the "less certain" ones presented. In our measurements we have resolved the individual decay channels.

There is good agreement between our work and the lifetimes calculated by Raasen \& Uylings (1997) for the $y^{4} \mathrm{~F}_{9 / 2,7 / 2}$ levels. However, there is a discrepancy between our lifetimes and the values of Raasen \& Uylings (1997) for $y^{4} \mathrm{~F}_{5 / 2,3 / 2}$ and the $z^{2} \mathrm{D}_{5 / 2,3 / 2}$. This may be due to a difference in the predicted level mixing, which is difficult to reproduce in semi-empirical calculations for complex atomic systems such as $\mathrm{Cr}$ II. In addition, an increase in the predicted level mixing may explain the discrepancy between our experimental $B F \mathrm{~s}$ and the calculated $B F$ s by Raasen \& Uylings (1997) for transitions from the $y^{4} \mathrm{~F}_{5 / 2,3 / 2}$ and the $z^{2} \mathrm{D}_{5 / 2,3 / 2}$ levels.

\subsection{Transition probabilities}

In Table 2 we present our $B F$ s and $A$-values. The $B F$ residual value in Table 2 is determined from the semi-empirical calculations of Raasen \& Uylings (1997). The residual value is used to estimate the $B F$ contribution from transitions that were not observed in our spectra. The missing lines are very weak transitions, $B F \leq 2 \%$, and for most upper levels in Table 2, the residual value is at most a few percent, which is less than the uncertainty in the $B F$ s. The uncertainty in the $A$-values is determined from the $B F$ and lifetime uncertainty using the method discussed 
Table 4. A comparison between our $A$-values and the $A$-values in the literature.

\begin{tabular}{|c|c|c|c|c|c|c|c|c|c|c|c|}
\hline \multirow{2}{*}{$\begin{array}{l}\text { Upper } \\
\text { level }\end{array}$} & \multirow{2}{*}{$\begin{array}{l}\text { Lower } \\
\text { level }\end{array}$} & \multirow{2}{*}{$\begin{array}{c}\sigma \\
\left(\mathrm{cm}^{-1}\right)\end{array}$} & \multicolumn{9}{|c|}{$A\left(10^{8} \mathrm{~s}^{-1}\right)$} \\
\hline & & & This study & $R \& U^{a}$ & $K^{b}$ & $W \& W^{c}$ & $G \& W^{d}$ & $M^{e}$ & $W t^{f}$ & $W e^{g}$ & $C \& B^{h}$ \\
\hline $3 \mathrm{~d}^{4}\left({ }^{3} \mathrm{H}\right) 4 \mathrm{p}^{4} \mathrm{H}_{13 / 2}^{\mathrm{o}}$ & $3 d^{4}\left({ }^{3} G\right) 4 s^{4} G_{11 / 2}$ & 30336.36 & 0.099 & 0.207 & 0.318 & & & 0.33 & 0.17 & $0.17^{i}$ & 0.86 \\
\hline $3 \mathrm{~d}^{4}\left({ }^{3} \mathrm{H}\right) 4 \mathrm{p}^{4} \mathrm{H}_{13 / 2}^{\mathrm{o}}$ & $3 \mathrm{~d}^{4}\left({ }^{3} \mathrm{H}\right) 4 \mathrm{~s}{ }^{4} \mathrm{H}_{13 / 2}$ & 33638.67 & 1.951 & 1.787 & 2.003 & & & 2.0 & 1.5 & $1.5^{i}$ & 5.1 \\
\hline $3 \mathrm{~d}^{4}\left({ }^{3} \mathrm{H}\right) 4 \mathrm{p}^{4} \mathrm{H}_{11 / 2}^{\mathrm{o}}$ & $3 \mathrm{~d}^{4}\left({ }^{3} \mathrm{H}\right) 4 \mathrm{~s}^{4} \mathrm{H}_{11 / 2}$ & 33550.22 & 1.851 & 1.672 & 1.804 & & & 1.9 & 1.6 & $1.7^{i}$ & 5.1 \\
\hline $3 \mathrm{~d}^{4}\left({ }^{3} \mathrm{H}\right) 4 \mathrm{p}^{4} \mathrm{H}_{9 / 2}^{\mathrm{o}}$ & $3 \mathrm{~d}^{4}\left({ }^{3} \mathrm{H}\right) 4 \mathrm{~s}^{4} \mathrm{H}_{9 / 2}$ & 33487.47 & 1.720 & 1.600 & 2.207 & & & 2.3 & 1.7 & $1.8^{i}$ & 3.6 \\
\hline $3 \mathrm{~d}^{4}\left({ }^{3} \mathrm{H}\right) 4 \mathrm{p}^{4} \mathrm{H}_{7 / 2}^{\mathrm{o}}$ & $3 \mathrm{~d}^{4}\left({ }^{3} \mathrm{H}\right) 4 \mathrm{~s}{ }^{4} \mathrm{H}_{7 / 2}$ & 33444.12 & 1.741 & 1.602 & 2.186 & & & 2.3 & 0.52 & $0.55^{i}$ & 6.1 \\
\hline $3 \mathrm{~d}^{4}\left({ }^{3} \mathrm{H}\right) 4 \mathrm{p}^{4} \mathrm{G}_{9 / 2}^{\mathrm{o}}$ & $3 \mathrm{~d}^{4}\left({ }^{3} \mathrm{H}\right) 4 \mathrm{~s}{ }^{4} \mathrm{H}_{9 / 2}$ & 37134.48 & 0.100 & 0.186 & 0.183 & & $0.13(7)$ & & & & \\
\hline $3 d^{4}\left({ }^{3} F\right) 4 p^{4} F_{5 / 2}^{o}$ & $3 \mathrm{~d}^{4}\left({ }^{1} \mathrm{D}\right) 4 \mathrm{~s}^{2} \mathrm{D}_{5 / 2}$ & 21281.50 & 0.011 & 0.006 & 0.007 & $0.046(9)$ & & & 0.0492 & & \\
\hline
\end{tabular}

Notes. ${ }^{(a)}$ Raasen \& Uylings (1997). ${ }^{(b)}$ Kurucz (1988). ${ }^{(c)}$ Wujec \& Weniger (1981). ${ }^{(d)}$ Goly \& Weniger (1980). ${ }^{(e)}$ Musielok \& Wujec (1979). ${ }^{(f)}$ Warner (1967) (Calculated). ${ }^{(g)}$ Warner (1967) (Experimental). ${ }^{(h)}$ Corliss \& Bozman (1962). ${ }^{(i)}$ Upper limit.

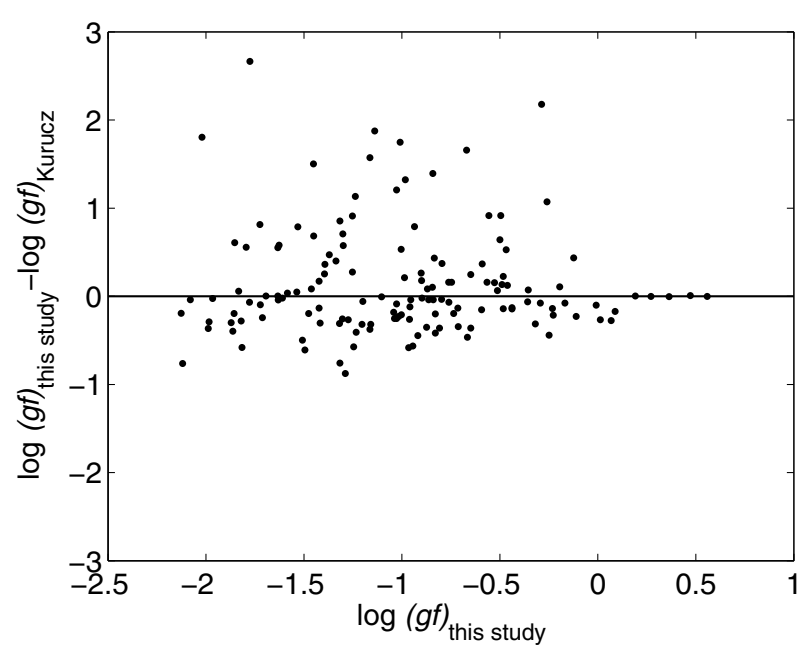

Fig. 3. Comparison between $\log (g f)$-values from this experimental study and semi-empirical values from Kurucz (1988). The difference between the two values for each line is plotted as a function of line strength.

by Sikström et al. (2002), which include uncertainties from the line-fitting, intensity calibration of each spectra, intensity cross calibration between separate spectra and the uncertainty in the fit of the decay curve.

The $\log g f$ s in Table 4 are compared to the semi-empirical results by Kurucz (1988) and Raasen \& Uylings (1997) and a graphical comparison is given in Figs. 3 and 4. The comparison between our laboratory $\log g f s$ and those of Raasen \& Uylings (1997), Fig. 4, has a smaller deviation than those in Fig. 3.

There is good agreement between our experimental log $g f$ values and the semi-empirical $\log g f$ values of Kurucz (1988) for lines from the $z^{4} \mathrm{H}_{J}$ levels as well as for lines from the $y^{4} \mathrm{G}_{5 / 2}$ and the $z^{2} \mathrm{D}_{3 / 2}$ levels, see Table 4 . However, there is a larger deviation between our $\log g f$ values and the semi-empirical calculations of Kurucz (1988) for lines from the $y^{4} \mathrm{~F}_{J}$ levels, the $y^{4} \mathrm{G}_{7 / 2,9 / 2,11 / 2}$ levels, and the $z^{2} \mathrm{D}_{5 / 2}$ level. This may be due to inaccurate level mixing in the semi-empirical calculations of Kurucz (1988). The semi-empirical calculations of Raasen \& Uylings (1997) agree more consistently with our laboratory $\log g f$ values.

In Table 4 we compare our $A$-values with $A$-values in the literature. In general our $A$-values agree to within the uncertainties with the results in the literature; however, the $A$ values of Corliss \& Bozman (1962) are significantly different to the other $A$-values in the literature. The compilation of

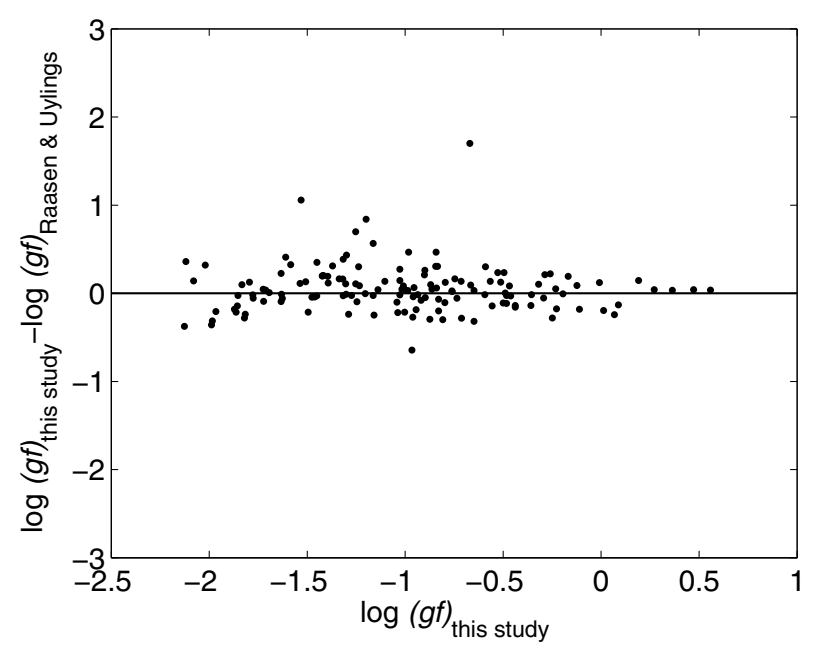

Fig. 4. Comparison between $\log (g f)$-values from this experimental study and semi-empirical values from Raasen \& Uylings (1997). The difference between the two values for each line is plotted as a function of line strength.

Corliss \& Bozman (1962) includes many values determined from a wall-stabilized arc. Inaccuracies in the wall temperature measurement for the wall-stabilized arc method can significantly increase the uncertainty in the measurement, so we recommend that the $A$-values in Corliss \& Bozman (1962) are used with caution.

\section{Summary}

We present experimental lifetimes for 14 highly excited energy levels in CrII and $B F$ s for 145 transitions from these levels, yielding experimental transition probabilities for lines that are strong features in stellar spectra. For the majority of the Cr II transitions in this paper, the experimental transition probabilities agree with the semi-empirical calculations within the uncertainty of the measurements. In particular, we note that the semi-empirical orthogonal operator calculations of Raasen \& Uylings (1997) have a one standard deviation difference to our experimental $\log g f$ values of 0.21 . However, the semi-empirical Cowan code calculations of Kurucz (1988) have a one standard deviation difference to our experimental $\log g f$ values of 0.61 . In addition, different semi-empirical calculations produce different term labels for the energy levels because of the large amount of level mixing. We suggested that further theoretical calculations for $\mathrm{Cr}$ II would benefit studies of this ion. 
J. Gurell et al.: The FERRUM project: laboratory-measured transition probabilities for Cr II

Acknowledgements. J.G. and S.M. are very grateful for the warm hospitality shown by the staff at Lund Observatory. Financial support from the Swedish Research Council (VR), a Linnaeus grant to the Lund Laser Centre and the Knut and Alice Wallenberg Foundation, is gratefully acknowledged. RBW acknowledges a Euopean Commision Marie Curie Intra-European fellowship.

\section{References}

Andrievsky, S. M., Kovtyukh, V. V., \& Usenko, A. 1994, A\&A, 281, 465 Babel, J., \& Lanz, T. 1992, A\&A, 263, 232

Bergeson, S. D., \& Lawler, J. E. 1993, ApJ, 408, 382

Bergström, H., Faris, H., Hallstadius, G. W., et al. 1988, Z. Phys. D 8, 17

Corliss, C. H., \& Bozman, W. R. 1962, Nat. Bur. Std. Monograph, 53

Dimitrijević, M. S., Ryabchikova, T., Simić, Popović, L. Č., et al. 2007, A\&A, 469, 681

Engman, B., Gaupp, A., Curtis, L. J., et al. 1975, Phys. Scr., 12, 220

Goly, A., \& Weniger, S. 1980, J. Quant. Spectrosc. Radiat. Transfer, 24, 335

Gonzalez, A. M., Ortiz, M., \& Campos, J. 1994, Can. J. Phys., 72, 57

Kurucz, R. L. 1988, Trans. IAU, XXB, ed. M. McNally (Dordrecht: Kluwer), 168

Lai, D. K., Bolte, M., Johnson, J. A., et al. 2008, ApJ, 681, 1524

López-García, Z., Adelman, S. J., \& Pintado, O. I. 2001, A\&A, 367, 859

Luke, T. M. 1988, J. Phys. B, 21, 4049

McWilliam, A., Preston, G. W., Sneden, C., et al. 1995, AJ, 109, 2757

Merrill, P. W. 1951, ApJ, 114, 37
Musielok, J., \& Wujec, T. 1979, A\&AS, 38, 119

Nave, G., Sansonetti, C. J., \& Griesmann, U. 1997, Optical Society of America Technical Digest, 3, 38

Nilsson, H., Ljung, G., Lundberg, H., et al. 2006, A\&A, 445, 1165

Pinnington, E. H., Lutz, H. O., \& Carriaveao, G. W. 1973, Nucl. Instrum. Methods, 110, 55

Pinnington, E. H., Guo, Q., J1., B., et al. 1993, Can. J. Phys., 71, 470

Raasen, A. J. J., \& Uylings, P. H. M. 1997, unpublished data available at: ftp: // ftp.wins.uva.nl/pub/orth/chromium

Ralchenko, Yu., Kramida, A. E., Reader, J., \& NIST ASD Team, 2009, NIST Atomic Spectra Database (version 3.1.5), [Online]. Available: http:// physics.nist.gov/asd3, October 12, National Institute of Standards and Technology, Gaithersburg, MD

Rice, J. B., \& Wehlau, W. H. 1994, A\&A, 291, 825

Schade, W., Mundt, B., \& Helbig, V. 1990, Phys. Rev. A, 42, 3

Shevchenko, V. S. 1994, Astron. Zh., 71, 572

Sikström, C. M., Nilsson, H., Litzén, U., Blom, A., \& Lundberg, H. 2002, J. Quant. Spectrosc. Radiat. Transfer, 74, 355

Sobeck, J. S., Lawler, J. E., \& Sneden, C. 2007, ApJ, 667, 1267

Spreger, R., Schelm, B., Kock, M., Neger, T., \& Ulbel, M. 1994, J. Quant. Spectrosc. Radiat. Transfer, 51, 779

Wallace, L., \& Hinkle, K. 2009, ApJ, 700, 720

Warner, B. 1967, Mem. Roy. Astron. Soc., 70, 165

Wujec, T., \& Weniger, S. 1981, J. Quant. Spectrosc. Radiat. Transfer, 25, 167

Xu, H. L., Svanberg, S., Quinet, P., Garnir, H. P., \& Biémont, É. 2003, J. Phys. B: At. Mol. Opt. Phys. 364773

Öberg, K. J. 2007, Eur. Phys. J. D, 41, 25 